\title{
Adverse Drug Reactions: Nitrosamine (and/or Telmisartan/HCT) Induced Nevus and Epidermal Scalp Cyst
}

\author{
Oliveira N, Kandathil LJ, Tchernev G* \\ Onkoderma, Policlinic for Dermatology, Venereology and Dermatologic Surgery, Sofia, Bulgaria
}

\begin{abstract}
Received: July 30, 2021; Accepted: August 01, 2021; Published: August 03, 2021
*Corresponding author: GeorgiTchernev, Onkoderma, Clinic for Dermatology, Venereology and Dermatologic Surgery, General Skobelev, E-mail: georgi_tchernev@yahoo.de
\end{abstract}

This is a presenting case of a 57 -year-old male with a longstanding history of primary arterial hypertension since the age of 13 years. He was placed on a treatment regimen of telmisartan/ hydrochlorothiazide (HTC) $80 / 12.5 \mathrm{mg}$ oral half tablets daily $(1 / 2-0-0) 10$ years ago and has continued with this treatment to this day. The patient recently visited our clinic due to growing concerns of an unclear and at the same time haemorrhagic lesion on the scalp (Figure 1a). According to the anamnestic data, the scalp lesion was thought to appear roughly 9-10 years after starting therapy with telmisartan/HTC and continued to grow in size to about $15 \times 8 \mathrm{~mm}$ (Figure 1a).

The patient is of Fitzpatrick phototype II and had no evidence of intense sun exposure till date. He was on no other medication apart from the aforementioned treatment for his hypertension which was well controlled. He was otherwise healthy, had no other concomitant conditions and family history regarding cutaneous cancers was negative. A preliminary biopsy from the lesion was conducted to rule out malignancy(Figure 1b).

The diagnosis of epidermal scalp cyst was made based on the histopathological findings of a unilocular cyst alongside intradermal nevi with pseudo-horn cysts that were detected (Figure 1e-h).

Due to the non-healing nature of the wound (Figure 1b), and the wish of the patient to prevent further bleeding, the lesion was surgically removed via elliptical excision under local anaesthesia and the primary wound closure was done with single interrupted sutures(Figure 1c-d).Postoperative findings showed poor signs of healing with the patient coming in for several check-ups which identified a new oval shaped lesion located in proximity to the prior excised lesion on the scalp. The histology has confirmed the diagnosis of intradermal nevus and epidermal scalp cyst again.

Therapy with so-called sartans or angiotensin receptor blockers could be extremely problematic. Contamination with certain nitrosamines could also be identified as one of the main reasons for this [1].

The reason for the manifestation or the establishment of certain stereotypes of manifestation of such side effects could be sought (except in the contamination, which probably occurred within the production process) as well as in the untimely adequate reaction of the control authorities with regard to checks whether certain batches of medicinal products are affected by nitrosamines or not [2].

And of course - in their subsequent rapid removal from the drug market. This is probably the reason for the higher frequency of the given side effects in certain geographical areas or those with reduced control [2].

Telmisartan or combination of telmisartan with hydrochlorothiazide have been reported and described in the scientific literature as possible inducers of nevus associated or occult melanomas, most likely due to possible contamination of the batches with nitrosamines [2,3].

The data for a significant association between the intake of sartans and melanoma date back to 2015/2016 and refer to groups of patients who develop melanoma and basal cell carcinoma after taking sartans or thiazide diuretics [4,5]. At that time, however, the topic of nitrosamine contamination and adverse drug reactions were not relevant.

Recently, valsartan has also been described as the first severe adverse drug reaction in Springer Nature / reactions weekly with respect to the occurrence of cutaneous melanoma, resulting in lethal outcome due to subsequent metastasis[1]. 

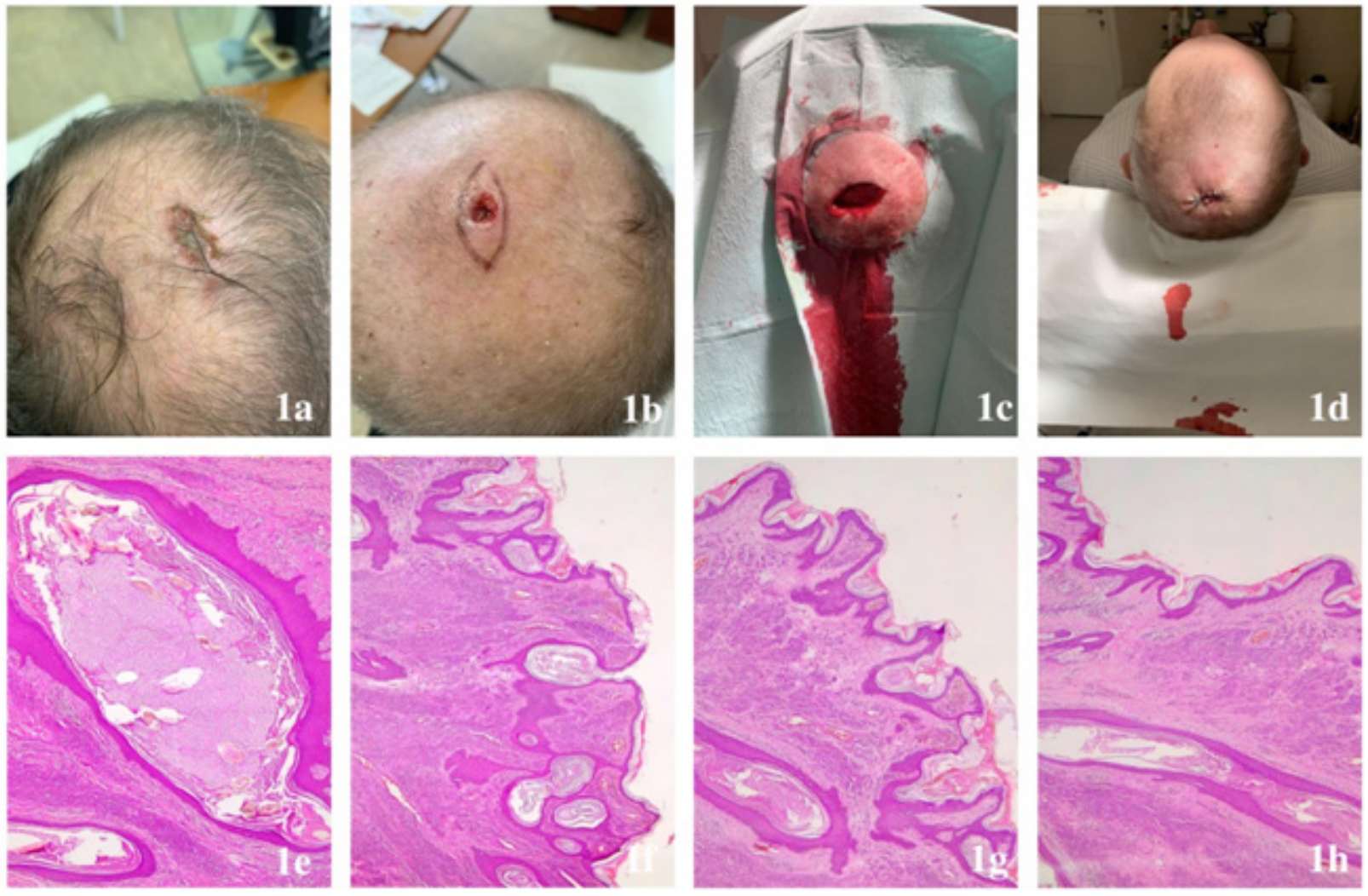

Figure 1a: Irregular pigmented cystic lesion measuring $15 \mathrm{~mm}$ x $8 \mathrm{~mm}$ located on the crown region of the scalp.

Figure 1b: Elliptical outlining of surgical margins after oval biopsy of the lesion.

Figure 1c: Intraoperative finding with elliptical excision of the lesion

Figure 1d: Postoperative finding with complete closure of defect with single interrupted sutures.

Figure 1e: Unilocular cystic lesion with dense granular keratinohyaline layer alongside hair shafts in the lumen (comedo). On the lower left side intradermal nevus.

Figure 1f: Intradermal nevus with pseudo-horn cysts.

Figure 1g-h: Intradermal nevus and a cystic dilatation of a hair follicle: an epidermoid cyst.

\section{References}

1. Tchernev G, Temelkova I. Valsartan Induced Melanoma?! First Description in Medical Literature! Open Access Maced J Med Sci. 2018;6(12):2378-2380.

2. Tchernev G, Bitolska A, Patterson JW. Telmisartan (and/or nitrosamine) - induced occult melanoma: first reported case in world literature. Expert Rev Clin Pharmacol. 2021;10:1-6.

3. Tchernev G, Patterson JW. Telmisartan/hydrochlorothiazide-induced nevus-associated cutaneous melanoma: first report in the medical literature. Expert Rev Clin Pharmacol. 2021;14(3):289-293.

4. Schmidt SA, Schmidt M, Mehnert F, Lemeshow S, Sørensen HT. Use of antihypertensive drugs and risk of skin cancer. J Eur Acad Dermatol Venereol. 2015;29(8):1545-1554.

5. Sable K, Majewski S, Nardone B. Association of melanoma and nonmelanoma skin cancer with antihypertensive drugs: A report from the Research on Adverse Drug events and Reports project. Poster 3167. J Am Acad Dermatol. 2016;74(5):AB221. 